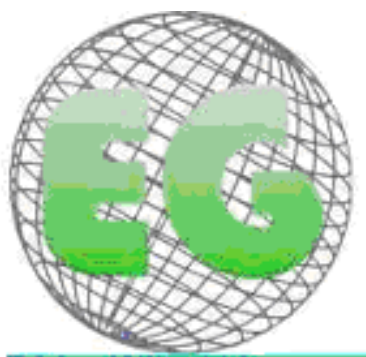

$\mathrm{N}^{\circ} 19$
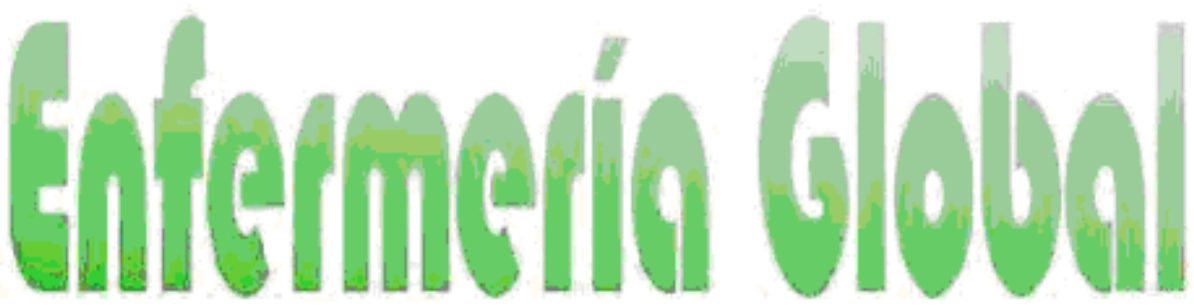

Reuista electrônica cuatrimestral de Enfermeria

Junio 2010

www.um.es/egloball

\title{
CLÍNICA
}

\section{PERFIL DE LOS CLIENTES DEL SISTEMA ÚNICO DE SALUD DE BRASIL (SUS) SOMETIDOS A IMPLANTE DE MARCAPASOS CARDÍACO DEFINITIVO EN HOSPITAL UNIVERSITARIO}

PEREIL DOS CLIENTES DO SUS SUBMETIDOS A IMPLANTE DE MARCAPASSO CARDÍACO DEFINITIVO EM HOSPITAL UNIVERSITÁRIO

*Lobo Domingo, EL. ${ }^{* *}$ Gonçalves Delatorre, P., ${ }^{* * *}$ Martins Rembold, S., ${ }^{* * * *}$ Lima da Silva, JL.

\begin{abstract}
* Pós-Graduando em Enfermagem na Promoção da Saúde da Universidade Federal Fluminense (UFF). **Pós-graduada em Enfermagem Gerontológica pela Universidade Federal Fluminense (UFF). *** Professora Adjunto do Departamento de Enfermagem Médico Cirúrgico da Escola de Enfermagem Aurora de Afonso Costa da Universidade Federal Fluminense (UFF). ${ }^{* * *}$ Mestre em Enfermagem pela Universidade do Rio de Janeiro (UNIRIO)- Brasil.

(Los datos de este trabajo fueron parcialmente presentados el día 6 de Julio de 2007 em el XIII Congreso SOLACI 2007 e XVII Congreso CACI 2007 en Argentina Buenos Aires bajo la forma de Palestra Oral intitulada Protocolos de Enfermagem para Implante de Marcapasso Cardíaco Definitivo.)
\end{abstract}

Palabras clave: aspectos sociodemográficos, marcapaso artificial, enfermería

Palavras chave: aspectos sócio-demográficos, marca-passo artificial, enfermagem

\section{RESUMEN}

El objetivo de este estudio fue investigar los aspectos sociodemográficos de los clientes atendidos en el Hospital Universitario (HU), de Rio de Janeiro/Brasil, para implante de marcapasos cardíaco definitivo entre diciembre de 2005 y diciembre de 2006. Se analizaron 78 historias clínicas, incluyendo todos los clientes sometidos a implante o cambio de marcapasos cardíaco definitivo en el sector de hemodinámica o en el centro quirúrgico del HU. De esos 78 clientes estudiados, 52 se sometieron a un implante de marcapasos y 26 al cambio de la unidad generadora. Se analizaron las siguientes variables: sexo, edad, sector de procedencia, diagnóstico principal, municipio de origen, tiempo de internación y forma de estimulación del marcapasos. De los 78 clientes estudiados, $66,67 \%$ eran de sexo femenino y $33,33 \%$ de sexo masculino. El promedio de edad de los clientes fue de 73,82 años. La indicación más común de implante de marcapasos cardíaco fue el bloqueo atrioventricular de tercer grado y el modo de estimulación del marcapaso fue el de cámara doble (DDDR), que permite la alteración de la frecuencia cardíaca, siempre y cuando sea necesario, a través de la estimulación de los atrios y ventrículos, de acuerdo con la frecuencia básica programada. 
Una vez realizado el implante del marcapasos, observamos una mejora significativa en la calidad de vida del cliente y la necesidad, por parte del equipo de enfermería, de enfatizar la importancia de los cambios en los hábitos de vida para el control de los factores de riesgo y la creación de grupos de apoyo.

\section{RESUMO}

O objetivo deste estudo foi levantar os aspectos sócio-demográficos dos pacientes atendidos no HUAP para implante de marcapasso cardíaco definitivo entre Dezembro de 2005 a Dezembro de 2006. Para tal foram analisados 78 prontuários compreendendo todos os pacientes submetidos a implante ou troca de marcapasso cardíaco definitivo no setor de hemodinâmica ou centro cirúrgico do HUAP. Desses 78 pacientes, 57 pacientes realizaram o implante de marcapasso e 17 a troca da unidade geradora. Foram analisados os seguintes parâmetros: sexo, idade, setor de procedência, diagnóstico principal, município de procedência, tempo de internação e o modo de estimulação do marcapasso. Do total de 78 pacientes, $68 \%$ eram do sexo feminino e $32 \%$ do sexo masculino, a média de idade dos pacientes foi de 73 anos. A indicação mais comum de implante de marcapasso cardíaco foi o bloqueio atrioventricular de $3^{\circ}$ grau e o modo de estimulação do marcapasso foi 0 DDDR. Após o implante de marcapasso observou-se uma melhora significativa na qualidade de vida do paciente e a necessidade de enfatizar mudanças nos hábitos de risco e a permanência em grupos de apoio.

\section{INTRODUCCIÓN}

Este artículo es el resultado de un desdoblamiento lineal del trabajo de monografía para la obtención del título de grado en enfermería titulado: Los cuidados de enfermería en los clientes sometidos a implante de marcapasos cardíaco definitivo: una propuesta para la elaboración de protocolos de atención ${ }^{[1]}$, realizada a partir de la observación de un número significativo de clientes hospitalizados en los hospitales públicos con cuadros de bradicardia y dolor precordial causado por arritmia cardíaca por bloqueo atrioventricular parcial o total, con indicación de implante de marcapasos (MP) cardíaco definitivo. Otro factor considerado para el abordaje del tema fue la constatación del déficit de conocimiento de los clientes con relación a su patología (señales y síntomas) y de los cuidados preventivos vinculados a las enfermedades cardiovasculares.

Según el Ministerio de Salud de Brasil (2006) [2:10]: "Las enfermedades circulatorias son responsables por un impacto expresivo en la mortalidad de la población brasileña, representando el $32 \%$ de los óbitos en 2002, el equivalente a 267.496 muertes. Las enfermedades del aparato circulatorio corresponden a un amplio espectro de síndromes clínicos, aunque las enfermedades relacionadas con la arterioesclerosis constituyen la causa principal que se manifiesta a través de enfermedades arteriales y coronarias, enfermedades cerebrovasculares de vasos periféricos, que incluyen patologías de la aorta, los riñones y los miembros, con morbosidad expresiva e impacto en la calidad de vida y la productividad de la población adulta”.

Actualmente en Brasil, las enfermedades cardiovasculares representan la principal causa de muerte, y ese cambio en el estándar de mortalidad no está solo determinado por el envejecimiento de la población sino, principalmente, por la persistencia de hábitos de alimentación y de actividad física inadecuados, además del tabaquismo. De acuerdo con el Ministerio de Salud de Brasil [2:11], los principales factores de riesgo de las enfermedades cardiovasculares son: Historia familiar de DAC prematura (familiar en primer grado de sexo masculino < 55 años y sexo femenino < 65 años; hombre > 45 años y mujer > 55 años; tabaquismo; hipercolesterolemia (LDL - c elevado); hipertensión arterial sistémica; diabetes 
melito; obesidad $\left(\mathrm{IMC}>30 \mathrm{~kg} / \mathrm{m}^{2}\right)$; adiposidad abdominal; sedentarismo; dieta pobre en frutas y vegetales; estrés psicosocial.

En algunos casos, las enfermedades cardiovasculares comprometen el funcionamiento del corazón y es necesario el implante de marcapasos. Las indicaciones de implante de marcapasos cardíaco definitivo en Brasil están definidas en la Normativa $\mathbf{n}^{\circ} 725$ del 6 de diciembre de 1999, publicada en el boletín oficial del 6/12/99 - Sección I [3], con base en los estudios realizados por la Sociedad Brasileña de Cirugía Cardiovascular/Departamento de Estimulación Cardíaca Artificial, consolidados en el Consenso DECA/SBCCV $1999^{[4]}$.

El Consenso DECA/SBCCV 1999 clasifica las indicaciones para la implantación de marcapaso definitivo o permanente en tres categorías:

CLASE I: Situaciones en las que existe acuerdo general en cuanto a la indicación del implante de marcapasos.

CLASE II: Situaciones en las que frecuentemente hay indicación de estimulación artificial, pero no hay acuerdo general relativo a su necesidad absoluta.

CLASE III: Situaciones en las que hay acuerdo general de que el implante de marcapasos no es necesario.

Las indicaciones de marcapasos definitivo o permanente relatadas en el Consenso DECA/SBCCV 1999 se clasifican de acuerdo con las siguientes categorías:

- Bloqueo atrioventricular de tercer grado (BATV);

- Bloqueo atrioventricular de segundo grado (BAV segundo grado);

- Bloqueo atrioventricular de primer grado (BAV primer grado);

- Bloqueos intraventriculares;

- Enfermedad del nudo sinusal;

- Síndromes neuromediados;

- Síncopes recurrentes de origen desconocido;

- Indicaciones especiales;

- Indicaciones para el cambio de generador;

- Indicaciones para el cambio de electrodo;

- Indicaciones para la reoperación con posibilidad de reposición o cambio de electrodo; Indicaciones especiales para el cambio de generador con cable electrodo.

Según el Ministerio de Salud de Brasil (2002) ${ }^{[5: 105]}$ : En los casos de emergencia por bradicardia severa FC < 40bpm, principalmente por bloqueo atrioventricular de segundo grado tipo II o bloqueo atrioventricular de tercer grado, el implante de marcapaso cardíaco definitivo sigue el algoritmo del protocolo de unidad de emergencia (ANEXO 1).

El problema de estudio de este artículo consiste en identificar los aspectos sociodemográficos que contribuyen a la determinación de enfermedades cardiovasculares significativas, como bloqueos atrioventriculares que pueden ocasionar el implante de marcapasos cardíaco definitivo. De esta forma, la delimitación del objeto de estudio permitió delinear algunas cuestiones para orientar la investigación: ¿Cuáles son los aspectos sociodemográficos de la clientela que inciden en el desenlace de las enfermedades 
cardiovasculares? ¿Cuáles son los tipos de estimulación de implante de marcapasos más utilizados? ¿Cómo es la calidad de vida de los clientes sometidos al implante?

Frente a las informaciones presentadas, el objetivo de este estudio es investigar los aspectos sociodemográficos de los clientes atendidos en el hospital universitario (HU) para el implante o cambio de marcapasos cardíaco definitivo entre diciembre de 2005 y diciembre de 2006. El HU, escenario de este estudio, es una institución pública de referencia para implantes de MP cardíacos definitivos del Sistema Único de Salud de Brasil (SUSB) para la región metropolitana II, integrada por siete municipios vecinos (Itaboraí, Maricá, Niterói, São Gonçalo, Tanguá, Rio Bonito y Silva Jardim) del Estado de Rio de Janeiro, Brasil.

El perfil de los clientes cardíacos usuarios de MP definitivo atendidos en el HU durante el período descrito fue delineado a partir de la investigación de historias clínicas, de los libros de registro de hemodinámica y de los registros presentes en la sala de revisión de implante de MP definitivo, con la finalidad de ofrecer información para la planificación de la atención de enfermería.

\section{JUSTIFICATIVA/ RELEVANCIA}

La investigación de los aspectos sociodemográficos de la clientela del hospital público universitario permitió el desarrollo de protocolos operacionales estándar, o planes de cuidados, con la definición de las acciones de enfermería para los clientes sometidos al implante de marcapasos cardíaco. También permitió acompañar el restablecimiento gradual de la salud de los clientes y su reintegración al contexto social en el que viven. De esta forma, la iniciativa del estudio contribuyó para el desarrollo de actividades de educación permanente en los sectores de la institución hospitalaria para el encaminamiento y la adecuación de esta nueva tecnología, elaborada por el equipo de enfermería, destinada a este sector de la clientela del servicio de salud.

\section{MATERIAL Y MÉTODOS}

La metodología utilizada consistió en el desarrollo de una investigación descriptiva, exploratoria y documental en el sector de hemodinámica del HU. Los sujetos seleccionados fueron los clientes atendidos en el sector de hemodinámica en el período comprendido entre diciembre de 2005 y diciembre de 2006. La recolección de datos se realizó a través de una investigación documental basada en las historias clínicas, libros de registro del sector y en los registros existentes en la sala de revisión de implante de MP definitivo, con la finalidad de ofrecer información para la planificación de la atención de enfermería.

De acuerdo con la resolución 196/1996 del Consejo Nacional de Salud (CNS), el estudio fue sometido al Comité de Ética en Investigación de la Facultad de Medicina del Hospital Universitario para su evaluación, siendo aprobado el 13/4/2007. El protocolo se encuentra disponible en el Sistema Nacional de Informaciones sobre Ética en Investigación incluyendo Seres Humanos (SISNEP) con el número de registro 0017.0.258.000-07.

Se analizaron todos los clientes de ambos sexos atendidos en el sector de hemodinámica del HU para implante o cambio de MP cardíaco definitivo entre diciembre de 2005 y diciembre de 2006. Los datos de los clientes recogidos durante el estudio se agruparon de acuerdo con las siguientes variables: sexo, edad, sector de procedencia, diagnóstico principal, municipio de origen, tiempo de internación y forma de estimulación del marcapaso (APÉNDICE 1). Los resultados se presentan en forma de gráficos y cuadros en valores porcentuales. 


\section{RESULTADOS}

Con relación a la distribución de los clientes en las variables sexo y edad, observamos el predominio de clientes de sexo femenino sometidos a implante de marcapasos cardíaco definitivo. De un total de 78 clientes, $66,67 \%$ eran de sexo femenino y $33,33 \%$ de sexo masculino. El promedio de edad de los clientes atendidos fue de 73,82 años (variando entre 36 y 95 años) con una predominancia mayor entre los 70 y los 90 años.

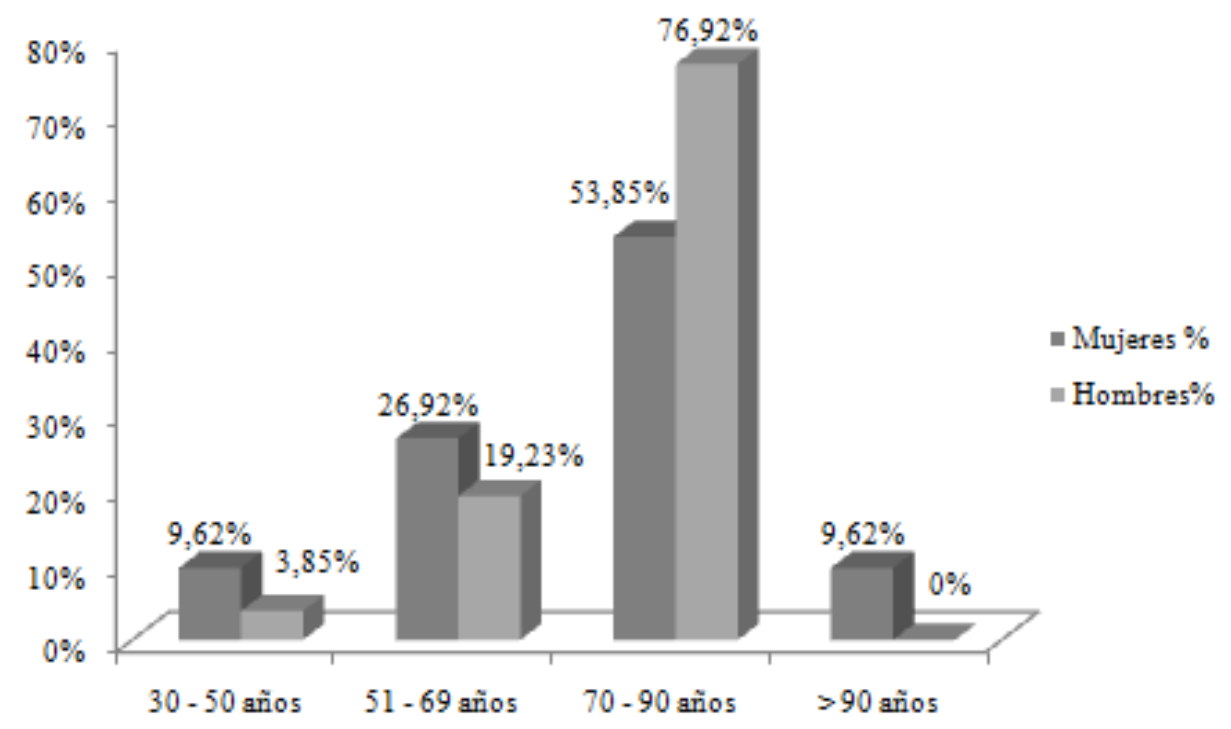

Figura 1: Distribución de las frecuencias en las variables sexo y edad de los clientes sometidos a implante de marcapaso cardíaco definitivo en el $\mathrm{HU}$, en el período comprendido entre diciembre de 2005 y diciembre de 2006.

Con relación a los sectores de procedencia de los clientes de las unidades de internación y de emergencia del HU y de las demás instituciones hospitalarias vecinas, puede observarse que la mayor parte de los clientes sometidos a implante de marcapasos cardíaco proviene de sectores del propio Hospital Universitario. La mayoría es oriunda de las clínicas médicas (masculina y femenina) $(39,7 \%)$, de su residencia (28\%) y de la unidad coronaria (UCO) $(21,7 \%)$. En frecuencias menores se observaron clientes provenientes del ala de reposo del HU $(3,38 \%)$ y aquellos oriundos de la atención por solicitud de procedimientos a través de la emisión de autorización para procedimientos de alta complejidad (APAC) por instituciones externas $(6,8 \%)$. 


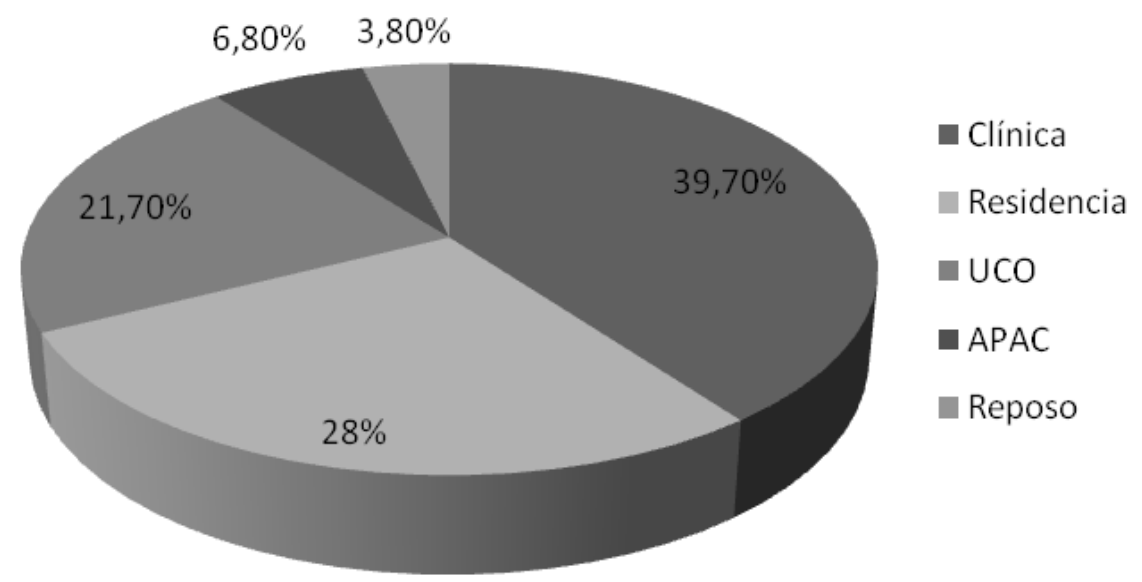

Figura 2: Distribución de las frecuencias en la variable sector de procedencia de los clientes sometidos a implante de marcapaso cardíaco definitivo en el $\mathrm{HU}$, en el período comprendido entre diciembre de 2005 y diciembre de 2006.

De los 78 clientes sometidos al procedimiento, 52 realizaron el primer implante de marcapasos, siendo que la mayoría de los clientes (32) de ambos sexos tenía una edad de entre 70 a 90 años. Los 26 clientes restantes cambiaron la unidad generadora o recibieron el implante de un nuevo marcapasos.

Cuadro 1. Distribución de las frecuencias de acuerdo con el número de implantes de marcapaso de los clientes sometidos a implante de marcapaso cardíaco definitivo en el HU, en el período comprendido entre diciembre de 2005 y diciembre de 2006.

\begin{tabular}{c|c|c}
\hline SEXO Y EDAD & IMPLANTE & REIMPLANTE \\
\hline Sexo femenino & & \\
30-50 años & 4 & 1 \\
51-69 años & 10 & 4 \\
70-90 años & 16 & 12 \\
> 90 años & 3 & 2 \\
Total & 33 & 19 \\
Sexo masculino & & \\
30-50 años & 1 & 0 \\
51-69 años & 2 & 3 \\
70-90 años & 16 & 4 \\
>90 años & 0 & 0 \\
Total & 19 & 7 \\
\hline
\end{tabular}

De los 26 clientes sometidos a reimplante, sólo cuatro (tres mujeres y un hombre) retornaron al HU para el reposicionamiento de los electrodos o de la unidad generadora debido a complicaciones relacionadas al uso del marcapasos. De los casos mencionados, se registraron dos casos de extrusión del marcapaso como fenómeno de rechazo. De acuerdo con St. Jude Medical ${ }^{[6: 11]}$, algunas de las posibles complicaciones relacionadas al uso del sistema de estimulación general son: embolia gaseosa; fenómenos de rechazo; taponamiento o perforación cardíaca; formación de tejido necrótico; falla en el funcionamiento del marcapaso; hematoma; migración del marcapaso; interferencia eléctrica e infección. 
Cuadro 2. Distribución de las frecuencias de acuerdo con el número de complicaciones relativas al uso de marcapaso de los clientes sometidos a implante de marcapaso cardíaco definitivo en el HU, en el período comprendido entre diciembre de 2005 y diciembre de 2006.

\begin{tabular}{c|c}
\hline SEXO Y EDAD & $\begin{array}{c}\text { REPOSICIONAMIEN } \\
\text { TO }\end{array}$ \\
\hline Sexo femenino & 0 \\
30-50 años & 2 \\
$51-69$ años & 0 \\
70-90 años & 1 \\
$>90$ años & 3 \\
Total & 0 \\
Sexo masculino & 1 \\
30-50 años & 0 \\
51-69 años & 0 \\
70-90 años & 1 \\
$>90$ años & \\
Total &
\end{tabular}

Con relación a la muestra de clientes relativa al diagnóstico principal antes del implante o cambio de marcapasos, se destacan: los bloqueos atrioventriculares en primero, segundo y quinto lugar (BAVT, BAV en segundo grado y BAV en primer grado) totalizando $67,9 \%$ de los diagnósticos. De este conjunto, el BAVT o bloqueo atrioventricular de tercer grado corresponde al $51,28 \%$ de las indicaciones. La fibrilación atrial con baja respuesta ventricular ocupa el tercer lugar y representa el $10,2 \%$ de los casos y en cuarto lugar se encuentran los diagnósticos desconocidos, que representan el 8,97\% de los clientes.

Cuadro 3: Distribución de las frecuencias con relación al diagnóstico principal de los clientes sometidos a implante de marcapaso cardíaco definitivo en el $\mathrm{HU}$, en el período comprendido entre diciembre de 2005 y diciembre del 2006.

\begin{tabular}{lcc}
\hline \multicolumn{1}{c}{ DIAGNÓSTICO } & N $^{\circ}$ DE & FRECUENCIA \\
\hline BAVT & 40 & 51,28 \\
BAV $2^{\circ}$ grado & 12 & 15,38 \\
Fibrilación atrial & 8 & 10,26 \\
Desconocido & 7 & 8,97 \\
BAV $1^{\circ}$ grado & 1 & 1,28 \\
Bradicardia sinusal / Mal & 1 & 1,28 \\
Enfermedad en el nudo & 1 & 1,28 \\
Hipersensibilidad del & 1 & 1,28 \\
IAM & 1 & 1,28 \\
Asincronismo AV & 1 & 1,28 \\
Falla de generador & 4 & 5,13 \\
Insuficiencia cardíaca & 1 & 1,28 \\
\hline
\end{tabular}

Según Smeltzer y Bare ${ }^{[7: 735]}$, las señales clínicas y los síntomas de bloqueo cardíaco varían con la frecuencia ventricular resultante y con la gravedad de cualquier proceso patológico subyacente. 
De acuerdo con Rembold y Domingos, et al (2008) [8:19]: "Los bloqueos atrioventriculares ocurren cuando algún disturbio agudo o crónico impide o dificulta el pasaje del estímulo eléctrico generado en el nudo sinusal al nudo atrioventricular. Esas alteraciones provocan cambios en el ritmo y en la frecuencia de contracción del miocardio, trayendo repercusiones hemodinámicas como la disminución del débito cardíaco."

El bloqueo AV de primer grado raramente causa algún efecto hemodinámico. Los otros bloqueos pueden resultar en la disminución de la frecuencia cardíaca, generando disminución en la perfusión de los órganos vitales como el cerebro, el corazón, los riñones, los pulmones y la piel. Un cliente con bloqueo AV de tercer grado provocado por intoxicación digitálica puede estar estable; otro cliente con el mismo ritmo causado por IAM puede estar inestable. El tratamiento de los bloqueos atrioventriculares se basa en el efecto hemodinámico provocado por la alteración en el ritmo cardíaco. Sin embargo, los profesionales de enfermería deben tener en cuenta que es necesario tratar al cliente con enfermedad cardiovascular de forma individualizada e integral, respetando su contexto histórico y social, y no se debe limitar al mantenimiento de un ritmo cardíaco estable.

Con relación a la distribución de los clientes sometidos a implante de marcapasos cardíaco definitivo, como podemos observar en los datos que figuran en el cuadro 3, la mayoría de los clientes son mujeres oriundas de Niterói, Municipio de Rio de Janeiro, donde se encuentra el HU, seguido de São Gonçalo y Rio de Janeiro, respectivamente. El número de clientes oriundos de municipios desconocidos corresponde a los clientes sometidos a implante de marcapaso cardíaco definitivo cuyas historias clínicas están incompletas, a los clientes atendidos en el servicio de emergencia o a través de APAC provenientes de otras instituciones hospitalarias, sin apertura de historias clínicas.

Cuadro 4: Distribución de las frecuencias según el municipio de residencia de los clientes sometidos a implante de marcapaso cardíaco definitivo en el $\mathrm{HU}$, en el período comprendido entre diciembre de 2005 y diciembre de 2006.

\begin{tabular}{lcccc}
\multicolumn{1}{c}{$\begin{array}{c}\text { MUNICIPIO DE } \\
\text { RESIDENCIA }\end{array}$} & $\begin{array}{c}\text { No } \\
\text { CLIENTES }\end{array}$ & $\begin{array}{c}\text { FRECUENCIA } \\
(\%)\end{array}$ & $\begin{array}{c}\text { HOMBRES } \\
(\%)\end{array}$ & $\begin{array}{c}\text { MUJERES } \\
(\%)\end{array}$ \\
\hline Niterói & 32 & 41,1 & 11,5 & 29,4 \\
São Gonçalo & 14 & 17,9 & 7,7 & 10,2 \\
Rio de Janeiro & 11 & 14,1 & 5,1 & 9,0 \\
Desconocido & 21 & 26,9 & 7,7 & 19,2 \\
\hline
\end{tabular}

Con relación a la cantidad de días de internación hospitalaria (DIH) (Cuadro 4), entre las 46 historias clínicas de los 78 clientes sometidos a este procedimiento, se observa que 32 no figuran en este cuadro debido a la falta de información registrada en 15 historias clínicas del HU. A eso se suma la existencia de 17 clientes sin historias clínicas (13 clientes registrados oriundos de su residencia y cuatro internados en otros hospitales) (Cuadro 4).

De las 46 historias clínicas analizadas, los clientes sometidos a implante de marcapasos cardíaco definitivo estuvieron internados un promedio de 9,63 días (1-34 días). Las mujeres estuvieron internadas por un período mayor que los hombres (34 DIH). Por su parte, los hombres presentaron un mayor tiempo promedio de internación (10 DIH). 
Cuadro 5: Distribución de las frecuencias de los días de internación hospitalaria (DIH), por sexo, de los clientes sometidos a implante de marcapasos cardíaco definitivo en el $\mathrm{HU}$, en el período comprendido entre diciembre de 2005 y diciembre de 2006.

\begin{tabular}{lccc}
\hline \multicolumn{1}{c}{ DIH } & $\begin{array}{c}\text { FEMENINO } \\
(\%)\end{array}$ & $\begin{array}{c}\text { MASCULINO } \\
(\%)\end{array}$ & $\begin{array}{c}\text { TOTAL DE CLIENTES } \\
(\%)\end{array}$ \\
\hline Máximo & 34,0 & 20,0 & 34,0 \\
Mínima & 1,0 & 1,0 & 1,0 \\
Promedio & 9,4 & 10,0 & 9,6 \\
Total de historias & & 15 & 46 \\
clínicas & 31 & & \\
\hline
\end{tabular}

Según Ramos, Ramos Filho y Rassi Junior et al (2003) [9:856]: "Los MP reciben códigos de cinco letras especificando las características de funcionamiento del aparato (cuadro 6). La primera letra se refiere a la cámara cardíaca estimulada por el electrodo. La segunda letra se refiere a la cámara de donde parte la actividad eléctrica sentida por el MP. La tercera al modo de respuesta del aparato después de captar o sentir la actividad eléctrica: inhibido (I), activado ( $T$ ) o ambos (D). A cuarta letra se refiere a los parámetros que pueden programarse: $\mathrm{R}$ es relativo a la frecuencia de respuesta; $C$ si el MP es capaz de recibir o transmitir datos; $\mathrm{M}$ indica que puede programarse en más de tres parámetros; $\mathrm{P}$ es limitado a menos de tres parámetros y $\mathrm{O}$, que es raramente encontrado, no es programable para ningún parámetro. La quinta letra se refiere a las características antidisrítmicas: $P$ caracteriza la actividad antitaquicárdica, $S$ se aplica a la función de cardioversión o de desfibrilación del MP y, finalmente, $\mathrm{D}$, normalmente la más observada, relativa a las dos posibilidades".

Cuadro 6: Código de cinco letras para los sistemas de marcapaso

\begin{tabular}{|c|c|c|c|c|}
\hline $\begin{array}{c}1 \text { 1a Letra } \\
\text { Cámara } \\
\text { Estimulada }\end{array}$ & $\begin{array}{l}2^{\circ} \text { Letra } \\
\text { Cámara } \\
\text { Sentida }\end{array}$ & $\begin{array}{c}\text { 3a Letra } \\
\text { Modo de } \\
\text { Respuesta } \\
\end{array}$ & $\begin{array}{c}4^{\text {a }} \text { Letra } \\
\text { Funciones } \\
\text { Programable } \\
\mathrm{s}\end{array}$ & $\begin{array}{c}5^{\text {a }} \text { Letra } \\
\text { Función anti } \\
\text { taquicardia }\end{array}$ \\
\hline $\mathrm{V}$ & $\mathrm{V}$ & $\mathrm{T}$ & $\mathrm{R}$ & 0 \\
\hline Z & $A$ & I & C & $\mathrm{P}^{* *}$ \\
\hline$D(A \& V)$ & $\mathrm{D}$ & $\mathrm{D}(\mathrm{T} / \mathrm{I})$ & $\mathrm{M}$ & S \\
\hline 0 & $\mathrm{O}$ & 0 & $P^{* *}$ & $D(P \& S)$ \\
\hline
\end{tabular}

$\mathrm{V}=$ Ventrículo; $\mathrm{A}=$ Atrio; $\mathrm{D}=$ Ambas posibilidades para la posición considerada; $\mathrm{O}=$ Ninguna; $\mathrm{T}$ = Activado; $\mathrm{I}=$ Inhibido; $\mathrm{R}=$ Frecuencia Modulada; $\mathrm{C}=$ Transmisión de datos; $\mathrm{M}=$ Multiprogramable; $\mathrm{P}^{*}=$ Simple Programable; $\mathrm{P}^{* *}=$ Antitaquicardia; $\mathrm{S}=$ Choque

Observamos que el $46,1 \%$ de los clientes de esta muestra contaban con programación DDDR (unidad reprogramable que reconoce el estímulo atrial y puede estimular los atrios y ventrículos); 30,7\% VVIR; 8,97\% VDDR; 8,97\% desconocido; 2,56\% SSIR; 1,28\% multiprogramable con telemetría y $1,28 \%$ resincronizador. Para controlar las alteraciones abruptas en el ritmo de los latidos cardíacos y mantener un buen débito sanguíneo para cada cliente, de acuerdo con cada diagnóstico, es necesario realizar una programación individual. A pesar de que muchas veces los modos de programación sean los mismos, las formas de estimulación son diferentes de acuerdo con algunos parámetros, entre otros, al inicio de la excitación de los atrios y de la resistencia de los ventrículos. 
Para Harrison, ${ }^{[10: 1064]}$ La estimulación de cámara doble (DDD) se indica para clientes que presentan los siguientes cuadros: enfermedad de nudo sinusal; bloqueo de AV crónico, sintomático, de $2^{\circ}$ y de $3^{\circ}$ grado; síndrome de Adams-Stokes recurrente; bloqueo bilateral de rama del haz, sintomático, cuando se asocia a la hipótesis de taquiarritmia y otras causas. La estimulación auricular se indica para clientes con alteración del nudo sinusal y sistema de conducción AV intraventricular normales. La estimulación puede modularse también por frecuencia fija o por demanda.

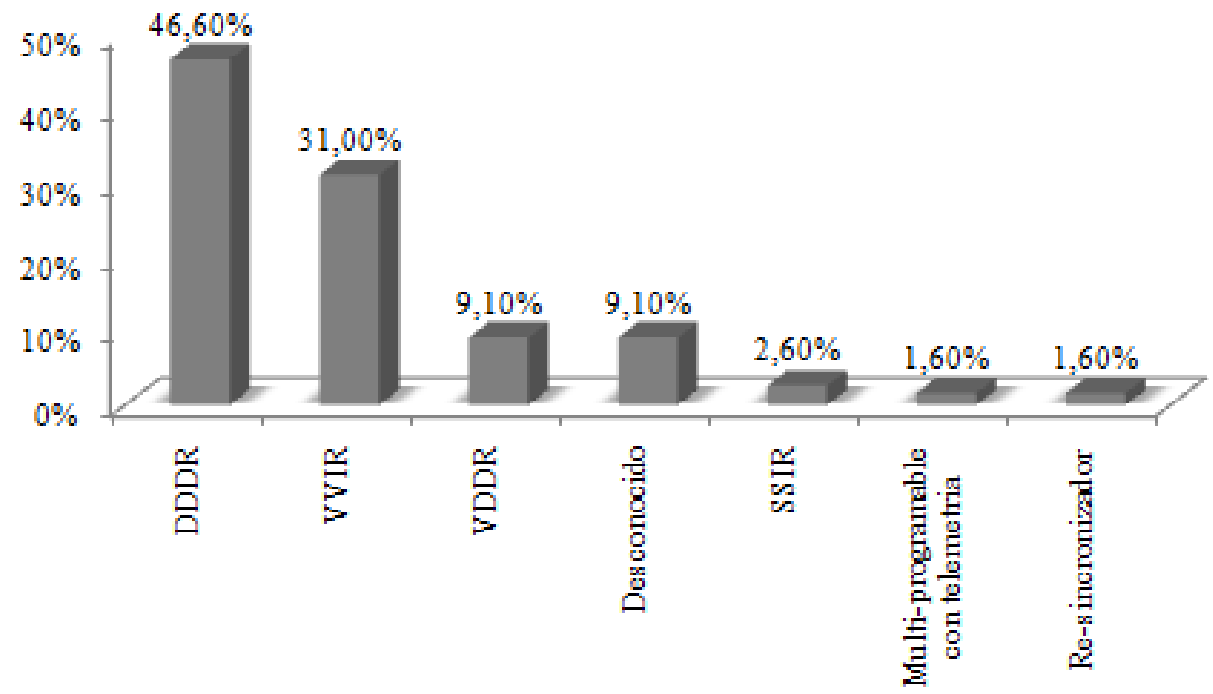

Figura 3: Distribución de las frecuencias según el modo de estimulación de los marcapasos implantados en los clientes sometidos a implante de marcapasos cardíaco definitivo en el $\mathrm{HU}$, en el período comprendido entre diciembre de 2005 y diciembre de 2006.

Este estudio, sumado a otros como el registro MAMI del año 2003 de la Sociedad Española de Medicina Intensiva, Crítica y de Unidades Coronarias ${ }^{[11]}$, permite concluir que los marcapasos DDD/DDDR verificaron un incremento progresivo en el número de implantes en los últimos años. Sin embargo, con relación al registro MAMI 2003 y el modo de estimulación de los marcapasos observados en los clientes del HU, presentan alteraciones significativas con relación al estudio español.

De acuerdo con los registros del MAMI 2003, los modos de estimulación del marcapasos presentan la siguiente disposición: VVI/VVIR 50\%, DDD/DDDR 24\%; VDD/VDDR 24\% y AAI/AAIR $2 \%$. A pesar de que en el presente estudio los modos de estimulación de todos los marcapasos de la muestra no han sido identificados debido a las fallas presentadas en los registros, el modo DDDR ocupa el primer lugar con el 46,1\%, y el VVIR en segundo lugar con el 30,7\%, seguido de VVIR 8,97\%, desconocido 8,97\%, SSIR 2,56\%, Multiprogramable con telemetría $1,28 \%$ y resincronizador, con $1,28 \%$.

Para Ochogavía y Baigorri ${ }^{[12]}$, a pesar de que el costo de implantación de los marcapasos cardíacos DDDR sea mayor en comparación al VVIR y del aumento en los riesgos de complicaciones postimplante, el modo de estimulación DDR puede considerarse el más próximo al fisiológico por mantener el ritmo sincrónico atrioventricular. 


\section{DISCUSIÓN}

El análisis de las historias clínicas se realizó a partir de los registros en los libros de implante de marcapasos en el sector de hemodinámica del HU y de la consulta directa de las historias clínicas de los clientes. Con relación a las variables evaluadas, resulta importante destacar que existieron dificultades para el establecimiento de los resultados debido a la falta de informaciones registradas en las fichas de algunos clientes. Para la realización de estudios vinculados a esa temática, es fundamental contar con historias clínicas completas para garantizar la precisión de la información.

Los resultados del presente estudio evidenciaron que la mayoría de los clientes con bloqueo atrioventriculares, principalmente de tercer grado, son sometidos a implante de marcapasos en el sector de hemodinámica del HU (68\%) y son de sexo femenino, al contrario de los estudios de Meltzer (1997) ${ }^{[13]}$, cuya mayoría era de sexo masculino. Según Meltzer (1997) [13:5-8], los principales factores de riesgo de las enfermedades cardíacas coronarias (DCC) son: hombres con edad superior a los 60 años, diabéticos; sedentarios; fumadores y adictos al tabaco, obesos y aquellas personas que presentan cuadros de hipercolesterolemia, principalmente tasas de low desinty lipoprotein (LDL) elevada, mantenimiento de niveles de presión iguales o superiores a 160/95 mm Hg, factores hereditarios para las DCC, estrés emocional y estándares de comportamiento agresivos, competitivos. De esta forma, las personas que presentan múltiples factores de riesgo son más propensas a desarrollar enfermedades cardíacas coronarias que aquellas que no los presentan.

Para Guyton e Hall, ${ }^{[14: 113]}$ cuando ocurre BAV de tercer grado, el impulso cardíaco generado en los atrios no pasa hacia los ventrículos por medio de los sistemas del nodo A-V y rama del haz $A-V$, los atrios continúan contrayéndose en la frecuencia rítmica normal del nodo sinusal, mientras el nuevo marcapaso se desarrolla en el sistema de Purkinje de los ventrículos, provocando la contracción ventricular en nueva frecuencia, cuyo valor permanece entre 15 a 40 latidos por minuto.

De esta forma, este posible cambio en el perfil de las personas con enfermedades cardíacas coronarias encontradas en esta muestra determinada por el predominio de mujeres con edad inferior a 65 años puede justificarse por el menor control de los factores de riesgo para las enfermedades cardiovasculares y la convivencia diaria con mayores niveles de estrés.

Para Meltzer ${ }^{[13]}$, el tratamiento conservador de la fase aguda del IAM consiste en la observación del cliente en la Unidad Coronaria (UCO), que acontece en los cinco primeros días después del infarto, y la fase subaguda del IAM abarca los días restantes de la hospitalización. El proceso de cicatrización del miocardio, frente a la ausencia de complicaciones serias, en general, tiene lugar en un tiempo de tres semanas de hospitalización, incluyendo el tiempo de internación en la UCO.

De esta forma, puede considerarse como tiempo de internación ideal, de forma conservadora, para los casos de implante de marcapasos cardíaco definitivo en situaciones de emergencia, para ambos sexos, entre un mínimo de 5 días y un máximo de 21 días El promedio de nuestra muestra fue de 9,6 días y el máximo de 34 días debido a complicaciones referentes a la extrusión del marcapaso y a la presencia de infecciones secundarias.

A partir de este estudio, también se puede observar una alteración significativa en los modos de estimulación del marcapasos que dejan de ser básicamente VVI estimulación de una única cámara, principalmente del ventrículo derecho, y pasan a DDD estimulación de las dos 
cámaras, atrio y ventrículo, considerado como modo de estimulación más cercano al estándar fisiológico para estimulación de las células cardíacas.

Para Ochogavía y Baigorri ${ }^{[12]}$, a pesar de que el costo de implantación de los marcapasos cardíacos DDDR sea mayor en comparación al VVIR y del aumento en los riesgos de complicaciones post implante, el modo de estimulación DDR puede considerarse próximo al fisiológico por mantener el ritmo sincrónico atrioventricular.

El modo de estimulación DDDR implica para el individuo una reducción en los riesgos de falla del marcapasos, síndrome del marcapasos y fibrilación atrioventricular. Sin embargo, no representa una reducción en los riesgos de accidente vascular cerebral (AVC) o una mejora significativa en la calidad de vida del cliente con el marcapasos implantado.

Contrariamente a los resultados obtenidos en la investigación de Ochogavía y Baigorri, para Brasil(2002 $)^{[15]}$ en una muestra de 80 clientes entrevistados inmediatamente antes y después del cuarto mes del implante de marcapasos cardíaco definitivo, sometidos a una evaluación de calidad de vida, utilizando el Índice de Calidad de Vida (IQV) de Ferrans y Powers: versión cardíaca (1992) traducido y adaptado para portadores de marcapasos, existe una diferencia entre la calidad de vida antes y después del implante del marcapasos definitivo: es mayor el Índice de Calidad de Vida después del implante $(14,88$ versus 17,43$)$.

De acuerdo con la teoría de la adaptación de Roy, y considerando que el implante de marcapasos definitivo puede ocasionar cambios físicos y emocionales significativos en el cliente, es necesario un proceso de adaptación del cliente a su nuevo estilo de vida. Por esa razón debe haber una interacción entre el enfermero y el cliente para poder aclarar aquello que dificulta el proceso de adaptación y trazar las acciones adecuadas (LEOPARDI, 1999) [16].

Para Moura y Silva(2005) ${ }^{[17]}$, el principal problema en el proceso de adaptación del cliente al implante de marcapasos es la falta de conocimiento o de comprensión relativa a los cambios ocurridos. Entre las alternativas para facilitar el proceso de adaptación y de adhesión al tratamiento y seguimiento de las orientaciones de enfermería, pensamos que la creación de grupos de apoyo es el instrumento más propicio para alcanzar esos objetivos de forma rápida y amena.

Durante el período de utilización del marcapasos permanente, según Domingos, Rembold y Delatorre (2008) ${ }^{[18]}$, el cliente portador del marcapaso y sus familiares deben estar atentos a las señales posibles de infección en el local del implante de marcapasos y a su funcionamiento adecuado. "Para eso, es necesario que el paciente realice una verificación periódica de la presión arterial, controle su frecuencia cardíaca y vaya regularmente a las consultas médicas (a los 10 días, 3 meses, 6 meses, de 6 en 6 meses) hasta que sea necesario realizar el cambio del generador del marcapasos" [18:5].

A pesar de los cambios registrados en la vida cotidiana, raramente el ambiente doméstico ocasiona problemas clínicos al portador del marcapasos. Si el ambiente doméstico cuenta con una red eléctrica con conexión a tierra y con aparatos en buen estado de conservación, es improbable que las interferencias electromagnéticas puedan ocasionar problemas en la vida cotidiana del cliente. Sin embargo, existen algunas restricciones vinculadas al ejercicio de algunas profesiones que exigen esfuerzo físico repetitivo o la convivencia con aparatos que generan fuertes campos magnéticos. 
A partir de los resultados de este estudio, pudieron observarse algunos aspectos o variables sociodemográficas de los clientes sometidos al implante de marcapasos cardíaco definitivo en el hospital universitario, lo que posibilitó trazar un plan de cuidados de enfermería adecuado al perfil de la clientela atendida en la Región Metropolitana II, facilitando las acciones del equipo en la atención.

De esta forma, con base en la consulta de historias clínicas y libros de registros de la hemodinámica, constatamos que las características del grupo de clientes usuarios de MP cardíaco definitivo internados o atendidos en los consultorios del HU fueron mujeres, ancianas con edad entre 70 y 90 años, oriundas de la clínica médica o de la unidad coronaria, con diagnóstico de bloqueo atrioventricular de tercero y/o segundo grado, residentes en el municipio de Niterói. Y observamos que el modo de programación de funcionamiento de la unidad generadora del marcapasos es el DDDR.

Pudo constatarse también un factor interesante: la presencia de un número significativo de personas portadoras de marcapasos con menos de 35 años.

Además, destacamos la importancia de la realización de estudios posteriores a partir de una muestra mayor compuesta por clientes del propio HU y de otros hospitales públicos o particulares de la región que realizan implante de marcapasos, ya que la comparación de estas informaciones podrá generar insumos necesarios para obtener una visión más amplia del perfil de la clientela atendida por el SUSB

Una vez realizado el implante del marcapasos, en el grupo que integra nuestro objeto de estudio, observamos una mejora significativa en la calidad de vida del cliente relacionada con la reducción de los síntomas anteriores al implante. Sin embargo, después del implante notamos algunas preocupaciones de los clientes vinculadas a las restricciones cotidianas y al miedo condicionado por la dependencia del marcapasos. Esta preocupación demuestra la necesidad de contar con equipos multidisciplinares, entre ellos de enfermería, que puedan enfatizar la importancia de los cambios en los hábitos de vida para el control de los factores de riesgo, favoreciendo el aumento de la calidad de vida de ese cliente en la etapa post implante. Para facilitar la adaptación del cliente a su nueva condición de salud y con el objetivo de reducir sus miedos y ansiedades, una alternativa viable surgida a partir de este estudio es la de integrarlo como coparticipante de su tratamiento a partir de la participación en reuniones de grupos de apoyo integrados por los clientes y sus familiares, que podrían realizarse en los días de consulta de revisión del marcapasos.

\section{REFERENCIAS}

1. DOMINGOS, E.L.L. Os cuidados de enfermagem aos clientes submetidos a implante de marcapasso cardíaco definitivo: uma proposta de elaboração de protocolo na assistência. (monografia) Niterói: EEAAC/UFF, 2007.

2. Brasil. Ministério da Saúde. Secretaria de Atenção à Saúde. Departamento de Atenção Básica. Prevenção clínica de doenças cardiovasculares, cerebrovasculares e renais / Ministério da Saúde, Secretaria de Atenção à Saúde, Departamento de Atenção Básica Brasília. Ministério da Saúde, 2006. 56p. - Cadernos de Atenção Básica; 14) ( Série A. Normas e Manuais Técnicos)

3. BRASIL. Indicações de implante de marcapasso cardíaco definitivo segundo Consenso DECA/SBCCV 1999. Portaria $\mathrm{n}^{\circ} 725$ de 06 de dezembro de 1999. Diário oficial de 06/12/99 - seção I. 
4. ANDRADE, J. C. S.; et al. Diretrizes para o implante de cardioversor desfibrilador implantável: Consenso DECA/SBCCV 1999. Rev Bras Cir Cardiovasc v.14, n.2, São José do Rio Preto, Abr. 1999.

5. Brasil. Ministério da Saúde. Protocolos da unidade de emergência/Hospital São Rafael Monte Tabor, Ministério da Saúde- 10 ed Brasília. Ministério da Saúde, 2002.

6. ST JUDE MEDICAL. Manual do utilizador dos geradores de impulsos Identity ${ }^{\top M} .2004$.

7. SMELTZER, S. C. ; BARE, B. G. Tratado de enfermagem médico-cirúrgica. $10^{a}$ ed. Rio de Janeiro: Guanabara Koogan, 2005.

8. REMBOLD, S. M.; DOMINGOS, E. L. L. ; DELATORRE, P. G.; LIMA, D.V.M.; TEIXEIRA, E.R. Protocolos de enfermagem: cuidados aos pacientes submetidos ao implante de marcapasso cardíaco definitivo. Enfermagem Brasil. Rio de Janeiro. Editora: Atlântica, $v$ $7, n 1$, jan/fev 2008.

9. RAMOS, G.; RAMOS FILHO, J.; RASSI JUNIOR, A.; PEREIRA, E.; GABRIEL NETO, S.; CHAVES, E.. Marcapasso cardíaco artificial: considerações pré e per-operatórias. Revista Brasileira de Anestesiologia, vol 53, n6, p. 854-862, Novembro-Dezembro, 2003.

10. HARRISON et al. Medicina interna. $13^{\text {a }}$ edição. Rio de Janeiro: Mc Graw Hill Interamericana, 1994, v. 1.

11. Sociedad Espanõla de Medicina Intensiva, Crítica y de Unidades Coronarias (SEMICYUC) [página principal en internet]. Registro MAMI 2003. Disponible en: $<$ http://www.semicyuc.org/sp/index-grupos-trabajo.htm>.

12. A. Ochagavia Calvo; F. Baigorri González. Selección del modo de estimulación del marcapasos. Medicina Intensiva. v. 30, n. 5, Madrid, Jun - Jul 2006.

13. MELTZER, L. E. Enfermagem na unidade coronária: bases, treinamento, prática. São Paulo: Editora Atheneu, 2001.

14. GUYTON, A.C. ; HALL, J. E. Fisiologia médica . 9a ed. Rio de Janeiro: Guanabara, 1998.

15. BRASIL, V. V. Qualidade de vida do portador de marcapasso cardíaco definitivo: antes e após implantes. Revista Eletrônica de Enfermagem (on-line), v. 4, n.1, p. 64, 2002. Disponível em <http://www.revistas.ufg.br/index.php/fen $>$.

16. LEOPARDI, M.T. Teorias em Enfermagem: instrumentos para a prática. Florianópolis: NFR/UFSC. 1999.

17. MOURA, R. C. ; SILVA, M. A. Cliente portador de marcapasso definitivo: a prática da adaptação. Livro-Temas do 56ํㅡㄹ Congresso Brasileiro de Enfermagem; Enfermagem hoje: coragem de experimentar muitos modos de ser [livro em formato eletrônico]; 2004 Out 24-29 [capturado 11 Jul de 2007]; Gramado (RS), Brasil. Brasília (DF): ABEn; 2005. Disponível em: http://bstorm.com.br/enfermagem.

18.DOMINGOS, E. L. L. ; REMBOLD, S. M.; DELATORRE, P. G. Os cuidados de enfermagem aos pacientes submetidos ao implante de marcapasso cardíaco definitivo: cartilha de orientação para a alta hospitalar. Rio de Janeiro. Editora: Fábrica do Livro, 2008. 


\section{APÉNDICE}

APENDICE I - FICHA UTILIZADA PARA LA INVESTIGACIÓN DE LAS HISTORIAS

\section{CLÍNICAS}

- Nombre

- $\quad \mathbf{N}^{\circ}$ de historia clínica:

- Fecha de nacimiento:

- Edad: Sexo: ( ) Masculino ( ) Femenino

- Diagnóstico principal:

- Lugar de residencia:

- Fecha de la internación hospitalaria:

- Fecha del implante de MP definitivo:

- Fecha de alta hospitalaria:

- Total de días de internación:

- ¿ ¿Hubo algún inconveniente relacionado al implante de marcapaso desde el período transoperatorio hasta la obtención del alta hospitalaria? （ ) Sí （） No

En caso afirmativo. ¿Cuáles?

- ¿Hubo algún inconveniente relacionado al implante de marcapaso en el período comprendido entre diciembre de 2005 y diciembre de 2006? （ ) Sí （） No

En caso afirmativo. ¿Cuáles?

- ¿Implantes anteriores? ( ) Sí ( ) No

En caso afirmativo. ¿Cuándo? 
VIII. ANEXO

ANEXO I - PROTOCOLO DE EMERGENCIA EN EL TRATAMIENTO DE BRADIARRITMIAS

\section{Bradiarritmia}

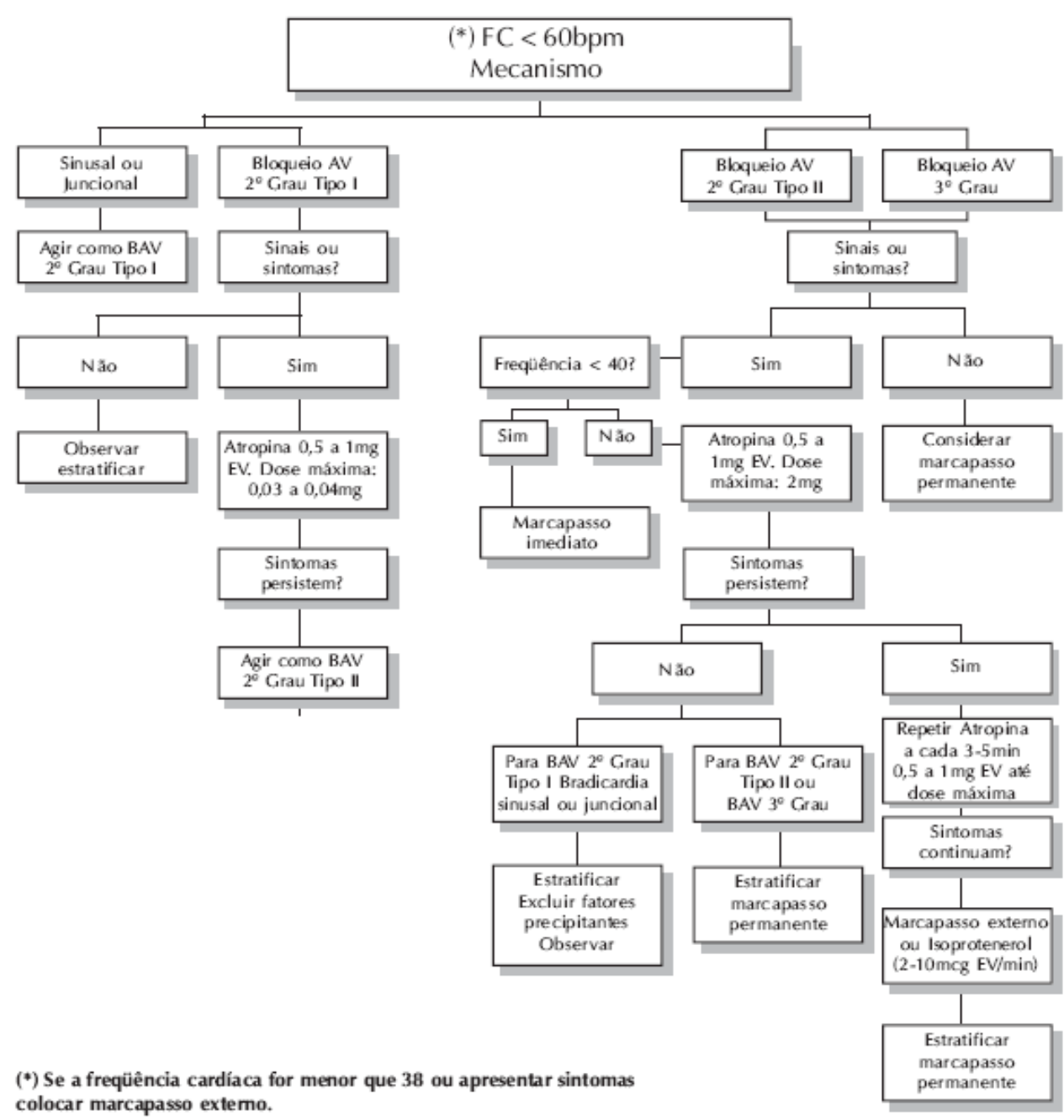

ISSN 1695-6141 